\title{
Satellite imagery of lengthy territories with complex configuration with the account of attitude and positioning errors
}

\author{
Dmitriy Mozgovoy ${ }^{1, *}$, Dmitriy Svinarenko ${ }^{1}$, Roman Tsarev $^{2}$, and Tatiana Yamskikh $^{2}$ \\ ${ }^{1}$ Oles Honchar Dnipropetrovsk National University, 72, Gagarin Prospect, Dnipropetrovsk, 49000, \\ Ukraine \\ ${ }^{2}$ Siberian Federal University, 79, Svobodny Prospect, Krasnoyarsk, 660041, Russia
}

\begin{abstract}
A method for monitoring attitude and positioning errors when taking satellite imagery of lengthy territories with complex configuration using an ultra-high spatial resolution optical-electronic scanner is described in the article. The results of modeling the system of automatic satellite attitude program control during the process of imagery are presented. Given these results, the impact of attitude and positioning errors during satellite imagery was estimated on the coverage percentage of the territory to be imaged.
\end{abstract}

\section{Introduction}

Satellite imagery is the most effective way to obtain spatial data due to its visibility, reliability, efficiency, contactless detailed observation and other advantages compared with traditional ground-based methods [1-6]. The new method of imagery in the process of retargeting (i.e., with non-zero angular velocities), implemented using satellite attitude program control, can significantly increase the efficiency and accuracy of satellite imagery of lengthy territories with complex configuration. This method of satellite imagery provides possibilities for:

- performing imagery of arbitrarily-spaced lengthy territories with complex configuration in one-orbit period (i.e., to increase the efficiency of imaging a given territory);

- increasing the amount of relevant remote sensing data obtained in one session (i.e., to increase the percentage coverage of a given territory);

- taking images from neighboring orbits at low angles of the Sun at a given signal-tonoise ratio;

- detecting low-contrast objects in the images under given lighting conditions;

- increasing the signal-to-noise ratio for satellite images and improve image classification procedures using radiometric and spectral characteristics.

Moreover, when taking satellite imagery in the retargeting mode, the duration of communication session in the direct transmission mode (for the same territory being

\footnotetext{
*Corresponding author: $\underline{\mathrm{m}-\mathrm{d}-\mathrm{k} @ \text { i.ua }}$
} 
imaged) increases, thus making it possible to reduce the data rate and use receiving stations with antennas having lower gain and smaller size (with the same parameters of onboard transmitting equipment ).

\section{Limitations of the proposed method}

The use of satellite attitude program control of a satellite angular motion imposes increased requirements on the on-board attitude system both in terms of providing the required attitude accuracy and stabilization of the satellite, and in providing the required retargeting velocity directly during the imagery. Moreover, special requirements apply to the subsystem of magnetic discharge of inertial energy-storage devices (flywheels). Therefore, there arose a necessity for computer modeling of new satellite imaging modes with ultrahigh spatial resolution scanners, given the attitude and positioning errors.

\section{The main stages of research}

The main stages of modeling satellite imagery of lengthy territories with complex configuration are as follows:

- approximation of a lengthy object defined by separate nodal points on a digital map (linear, quadratic, spline, etc.);

- determination of the optimal coverage of a lengthy object, taking into account the swath width of the imaging instrument and the required scanning direction;

- calculation of the orbital motion in order to choose the orbit for imagery and the time for switching on the imaging instrument, taking into account the Delay coefficient (when implementing the "Time Delay and Integration" mode);

- calculation of the satellite attitude angles required for imaging a given lengthy object, taking into account the installation angles of the imaging instrument;

- estimation of the satellite angular velocities during the imagery and analysis of the imagery feasibility, taking into account the existing limitations on the values of pointing angles and satellite angular velocities;

- modeling of satellite imagery process, taking into account additional factors (atmospheric refraction, terrain relief displacement, etc.);

- estimation of the impact of systematic and random attitude and positioning errors on agility and coverage percentage.

\section{The description of mathematical models used}

\subsection{Mathematical model of a lengthy territory with complex configuration}

The territory with complex configuration to be imaged is marked on the map by nodal points with arbitrary step in latitude and longitude. To approximate the lengthy territory, a natural cubic interpolating spline is used, then smoothed with the least squares method.

\subsection{Determination of the required satellite attitude in the process of imagery}

The input data are composed of:

- coordinates of the reference points in geographic coordinate system corresponding to start and end points of the line;

- satellite coordinates in geographic coordinate system; 
- pointing vectors of the reference points in the instrument coordinate system;

- the values of the elements in the transition matrix from the orbital coordinate system to the geographic coordinate system.

Yaw, pitch and roll attitude angles and angular velocities identify a reference frame that moves with the satellite.

The calculation procedure is as follows:

- normalised vectors of the reference points alignment in geographic coordinate system are calculated;

- the values of the elements in the transition matrix from the geographic coordinate system to the orbital coordinate system are determined;

- vectors of the reference points alignment in the orbital coordinate system are calculated;

- the values of the elements in the transition matrix from the instrument coordinate system to the orbital coordinate system are defined;

- yaw, pitch and roll attitude angles are determined by the values of the elements in the transition matrix from the instrument coordinate system to the orbital coordinate system.

\subsection{The model of satellite orbital motion}

For short-term prediction and low earth circular orbits, sufficient accuracy is achieved with the propagator SGP4, which allows to work with the initial conditions in TLE files generated by NORAD.

\subsection{Review and analysis of the impact of satellite attitude and positioning errors}

The impact of random errors that occur when setting attitude parameters and satellite tracking on the feasibility and efficacy of the imagery program in terms of the coverage accuracy was estimated using the finite differences method.

The input data are composed of the errors in specifying the attitude angles and prediction of the satellite position. The output data are the displacements of the reference points' cartographic coordinates corresponding to the maximum attitude errors and the position of the satellite.

The peculiarity of the method consists in calculating the values of partial differentials from the reference points' cartographic coordinates in the satellite coordinates and the roll yaw and pitch angles. They are calculated for all combinations of maximum and minimum absolute values of errors in specifying satellite attitude angles and prediction of the satellite position corresponding to start and end points of all lines in the scene being imaged.

\section{The input data for modeling}

The input data for modeling satellite imagery of lengthy territories with complex configuration are as follows:

- the territory to be imaged is the southern coast of Crimea from Sevastopol to Feodosiya;

- the type of satellite orbit - solar-synchronous,

- the altitude of the satellite orbit is $700 \mathrm{~km}$;

- the scanner swath width at nadir - $20 \mathrm{~km}$.

As a basis for modeling we used the type of imagery without TDI (Time Delay and Integration) implementation when satellite attitude errors were not considered in 
simulation, as well as with the maximum satellite attitude errors $\pm 0.1^{\circ}$ in roll, pitch and yaw.

\section{The results of modeling}

The results of modeling satellite imagery of lengthy territories with complex configuration (the scanner swath width $-20 \mathrm{~km}$ ) when satellite attitude errors were not considered are shown in Figure 1. Figure 2 presents the results of modelling with the maximum satellite attitude errors.

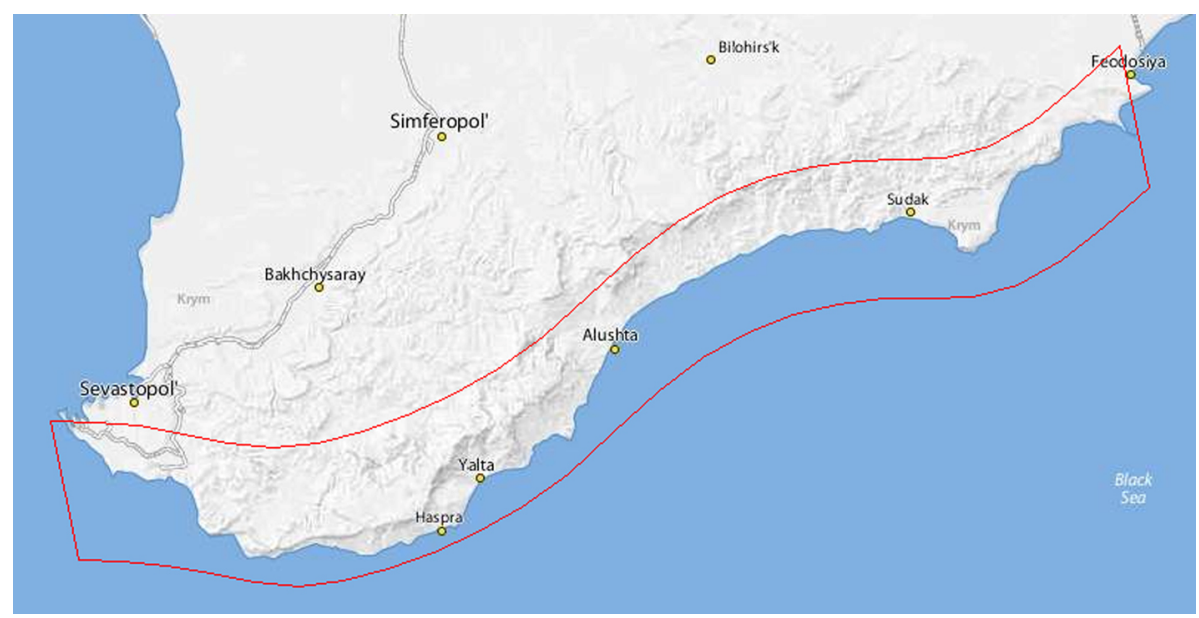

Fig. 1. Modeling satellite imagery when satellite attitude errors are not taken into account.

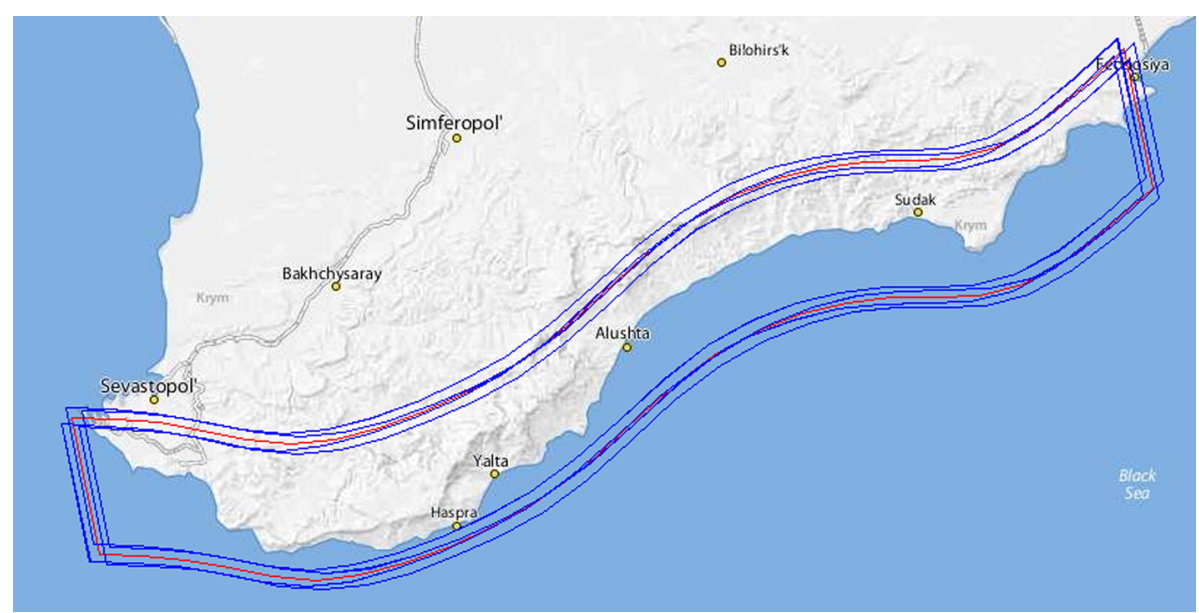

Fig. 2. Modeling satellite imagery with the account of the maximum satellite attitude errors $\pm 0.1^{\circ}$ in roll, pitch and yaw.

The simulation results of satellite imagery of the lengthy coastline on the southern coast of Crimea from Sevastopol to Feodosiya with measurement data of cloud amount for a given area showed, that in a given 5-day period, the territory to be imaged was completely available for 1 day, partially available for 2 days, unavailable for 2 days. 
The results of simulating the impact of satellite attitude and positioning errors when scanning a lengthy coastline showed that with a maximum satellite attitude error of $\pm 0.1^{\circ}$ for roll, pitch and yaw, about $90 \%$ and $80 \%$ of the territory was covered with the scanner swath width 40 and $20 \mathrm{~km}$ respectively. The satellite orbit prediction errors did not reduce the percentage of guaranteed coverage due to their insignificant values (sub-pixel mapping was used for a given scale).

\section{Evaluation of the method for satellite imagery of lengthy territories with complex configuration}

Table 1 represents the parameters determining the efficiency of modeling for various types of satellite imagery of the lengthy coastline on the southern coast of Crimea from Sevastopol to Feodosiya taking into account measurement data of cloud amount for a given area and satellite attitude and positioning errors.

Table 1. The basic parameters determining the efficiency of modeling for various types of satellite imagery taking into account measurement data of cloud amount for a given area and satellite attitude and positioning errors

\begin{tabular}{|l|c|c|c|}
\hline \multicolumn{1}{|c|}{ Type } & $\begin{array}{c}\text { The number } \\
\text { of scenes } \\
\text { (orbits) }\end{array}$ & $\begin{array}{c}\text { Task } \\
\text { duration, } \\
\text { days }\end{array}$ & Coverage \\
\hline $\begin{array}{l}\text { Imagery with scene orientation along } \\
\text { the subsatellite track direction }\end{array}$ & 3 & 5 & $52 \%$ \\
\hline $\begin{array}{l}\text { Imagery with an arbitrary scene } \\
\text { orientation }\end{array}$ & 1 & 1 & $80 \%$ \\
\hline $\begin{array}{l}\text { Imagery of scenes with complex } \\
\text { configuration }\end{array}$ & 1 & 1 & $98 \%$ \\
\hline
\end{tabular}

In the process of satellite imagery of lengthy territories with complex configuration, the new method gave an increase in the effective area of about $15 \%$. Weather conditions are more important for autumn-winter period. Thus, the comparative efficiency of the method developed for satellite imagery will be even higher. Improving the efficiency of imagery by means of satellite attitude program control during imagery provides possibilities for:

- reducing the time required for taking images of arbitrarily-spaced lengthy territories with complex configuration;

- increasing the signal-to-noise ratio of satellite images when taking images from neighboring orbits at low angles of the Sun;

- increasing the amount of relevant remote sensing data obtained in one session.

\section{The technique for estimating requirements to attitude and stabilization subsystems of remote sensing satellites}

On the basis of the developed mathematical models, we developed a technique for estimating requirements to attitude and stabilization subsystems of remote sensing satellites with optical-electronic scanners of ultrahigh spatial resolution, which increases the efficiency and reliability of data obtained while scanning small-sized objects located on lengthy territories with complex configuration. Based on the requirements to the agility and the percentage coverage of the lengthy territory to be imaged, this technique provides possibilities for the development of requirements to attitude and stabilization subsystems of 
remote sensing satellites with optical-electronic scanners of ultrahigh spatial resolution at the design stage.

\section{Conclusion}

The results of modeling satellite imagery of lengthy territories with complex configuration, with the account of satellite attitude and positioning errors, confirmed the efficiency of automatic satellite attitude program control during the process of imagery.

Simulated satellite imagery of the lengthy coastline on the southern coast of Crimea from Sevastopol to Feodosiya with the account of measurement data of cloud amount for a given area and satellite attitude and positioning errors, demonstrated high agility of the method proposed (1 day) and higher percentage of the effective area (98\%) compared to traditional satellite imagery ( 5 days and $52 \%$ respectively).

The series of simulation results allowed us to conclude that one-orbit imagery of a scene characterized by complex configuration using satellite attitude program control during the process of imagery is the most effective type of satellite imagery in terms of agility and coverage percentage.

\section{References}

1. P.C. Gray, J.T. Ridge, S.K. Poulin, A.C. Seymour, A.M. Schwantes, J.J. Swenson, D.W. Johnston, Remote Sens., 10, 1257 (2018)

2. X. Huang, D. Wen, J. Li, R. Qin, Remote Sens. Environ., 196, 56-75 (2017)

3. Y. Kim, S.B. Lee, H. Yun, J. Kim, Y. Park, Int. Geoscience and Remote Sensing Symposium (IGARSS), 4862-4865 (2017)

4. J. Liu, W.J. Emery, X. Wu, M. Li, C. Li, L. Zhang, IEEE T. Geosci. Remote, 55, 71137125 (2017)

5. M.S. Moussavi, W. Abdalati, A. Pope, T. Scambos, M. Tedesco, M. MacFerrin, S. Grigsby, Remote Sens. Environ., 183, 294-303 (2016)

6. O.L. Makarov, D.K. Mozgovoy, V.S. Khoroshilov, 21th Int. Conf. on Microwave and Telecommunication Technology (CriMiCo), 905-907 (2011) 